\title{
MELAS Syndrome
}

National Cancer Institute

\section{Source}

National Cancer Institute. MELAS Syndrome. NCI Thesaurus. Code C84885.

A rare progressive neurodeg enerative disorder characterized by mitochondrial myopathy, encephalopathy, lactic acidosis, and stroke-like episodes. 